\title{
Age influence on renalase and catecholamines concentration in hypertensive patients, including maintained dialysis
}

This article was published in the following Dove Press journal:

Clinical Interventions in Aging

28 October 2016

Number of times this article has been viewed

\section{Edyta Zbroch \\ Dominika Musialowska \\ Ewa Koc-Zorawska \\ Jolanta Malyszko}

Second Department of Nephrology and Hypertension with Dialysis

Centre, Medical University of

Bialystok, Bialystok, Poland
Correspondence: Edyta Zbroch Second Department of Nephrology and Hypertension with Dialysis Centre, Medical University of Bialystok, 15-276 Bialystok, Sklodowskiej Curie 24a, Poland Tel/fax +48 85 83। 7872

Email edzbroch@poczta.onet.pl
Background: Hypertension in elderly patients is one of the main problems in cardiovascular diseases. The sympathetic nervous system hyperactivity seen in older patients is a known risk factor for hypertension and other cardiovascular events as well as chronic kidney disease. Renalase, secreted by the kidney and circulated in blood, may regulate the sympathetic tone by catecholamine degradation and in this way has an impact on cardiovascular and renal complications.

Objective: To assess the impact of age on renalase and catecholamine concentration in hypertensive patients, including those on dialyses and its possible relation to blood pressure control and cardiovascular disease.

Methods: The study cohort of 211 patients was divided into two groups according to age below 65 years (range 19-64) and above 65 years (range 65-86). The older group represented 38\% of the whole studied population and $75 \%$ of them were dialyzed. The two groups of different ages were also divided into dialysis and nondialysis subgroups. The serum renalase, dopamine, and norepinephrine concentration together with blood pressure value and echocardiography were assessed.

Results: Patients aged 65 years and more had higher renalase $(20.59 \mathrm{vs} 13.14 \mu \mathrm{g} / \mathrm{mL}, P=0.02)$ and dopamine ( $41.71 \mathrm{vs} 15.46 \mathrm{pg} / \mathrm{mL}, P<0.001)$ concentration as well as lower diastolic blood pressure ( 75.33 vs $85 \mathrm{mmHg}, P=0.001$ ), advanced abnormalities in echocardiography, and more often suffered from diabetes and coronary artery disease. The significant correlation between age and renalase $(r=0.16 ; P=0.019)$, norepinephrine $(r=0.179 ; P=0.013)$, and dopamine $(r=0.21$; $P=0.003$ ) was found in the whole study population. In the nondialysis subgroup, $44 \%$ had chronic kidney disease, mostly in the stage $2(83 \%)$. There was a significantly higher norepinephrine concentration ( $1.21 \mathrm{vs} 0.87 \mathrm{ng} / \mathrm{mL} ; P=0.008)$ in older patients of that population. In the dialysis subgroup, there were no differences between renalase and catecholamine level but older participants had lower diastolic blood pressure ( 69 vs $78 \mathrm{mmHg}, P=0.001$ ) and ejection fraction ( $51 \%$ vs $56.8 \%, P=0.03)$.

Conclusion: The elevated renalase level in older hypertensive patients is related rather to kidney function and cardiovascular diseases than to age itself. Thus, renalase appears to be the possible new marker of these indications in this special population.

Keywords: renalase, catecholamines, older age, hypertension, cardiovascular complications, chronic kidney disease

\section{Introduction}

The world population is increasingly aging. Hypertension in elderly patients is one of the main problems in cardiovascular diseases (CVDs). ${ }^{1}$ Older hypertensive adults experience cardiovascular events at a rate two to three times greater than the younger 
with the same blood pressure (BP) values. ${ }^{2}$ According to recent data, patients aged over 65 years more often suffer from chronic kidney disease (CKD). ${ }^{3} \mathrm{CKD}$ as well as older age is characterized by elevated sympathetic nerves system activity, which correlates with increased vascular resistance and systemic BP and leads to CVD. Sympathetic nerves system overactivity in renal patients also aggravates kidney failure progression. ${ }^{4-6}$

The year 2005 brought the discovery of renalase - a protein, released by kidney, which may take part in the catecholamines degradation and result hemodynamic effect. ${ }^{7}$ Renalase infusion in rats caused a dose-dependent decrease in cardiac contraction, heart rate, and BP and prevented a compensatory increase in peripheral vascular tone. Using the Western blot test, the researchers from Yale School of Medicine showed serum renalase deficiency in patients with CKD treated with hemodialysis (HD) compared to healthy individuals. ${ }^{8}$ Renalase deficiency was also found in rats subjected to subtotal nephrectomy. ${ }^{9}$ A recent animal study proved the protective renalase role in the progression of cardiorenal syndrome. ${ }^{10}$ On the other hand, clinical studies using the enzyme-linked immunosorbent assay (ELISA) test showed a higher renalase level in patients with CKD and kidney transplant recipients compared to healthy volunteers. ${ }^{1-16}$ Some of the genetic studies confirmed the connection between renalase and hypertension as well as CVDs. ${ }^{17-19}$ But, there are also the opposite investigations..$^{20,21}$

The aim of our study was to assess, for the very first time, the age influence on renalase and catecholamine concentration in hypertensive patients, including maintained dialysis, and to find out if the suspected impact depends on BP control and CVD.

\section{Methods}

We involved in the study 211 patients with hypertension (53\% male) in the mean age of 58 years (19-86), including maintained HD and peritoneal dialysis (PD) (mean time on renal replacement therapy - 27 months). Patients were recruited from the Hypertension Outpatient Unit (HT) and the Dialysis Center in Bialystok, Poland, from November 2011 to January 2013. The inclusion criteria contained the diagnosis of hypertension made according to medical records or the fact of hypotension therapy. All patients without the history of hypertension were excluded. The study cohort was divided into two groups - according to age below 65 years (range 19-64) and above 65 years (range 65-86). The two groups of different age were also divided into dialysis and nondialysis subgroups. The serum renalase, dopamine, and norepinephrine concentration were assessed as well as BP rate, body mass index, laboratory tests, and echocardiography.

All patients were informed about the aim of the study and provided written informed consent. The study was approved by the Medical University Ethic Committee. The clinical trial registration number is: R-I-002/238/2011. BP was measured in the HD group - before and after HD session and in HT and PD patients - during routine ambulatory control, in the sitting position using automatic manometer. The arithmetic average of three measurements taken in the different days was used in the analysis. The well-controlled BP was assessed according to European Society of Cardiology/European Society of Hypertension and Kidney Disease Outcomes Quality Initiative guideline as lower than 140/90 $\mathrm{mmHg}$ in HT and PD patients and in HD patients before HD session and lower than $130 / 80 \mathrm{mmHg}$ after that. ${ }^{22-24}$ Body mass index was calculated according to the dry weight. The kind of hypotensive drugs was collected from the individual prescription cards. The blood for the estimation of the renalase, catecholamine concentration, and laboratory tests was taken once, in the HT and PD groups - while routine ambulatory visit and in HD group - before the HD session in the middle of three dialysis sessions (when also BP, heart rate, and weight were assessed). The ELISA kit made by Uscn Life Science Inc. China, using a monoclonal antibody specific to renalase, was taken to assess renalase level. The same method was used to assess catecholamine level - Noradrenaline ELISA kit and Dopamine ELISA kit - both made by Labor Diagnostica Nord GmbH \& Co. KG, Germany. Laboratory tests were estimated using standard methods in the central laboratory.

\section{Statistical analysis}

The data were analyzed using Statistica 10 computer software (Tulsa, OK, USA). The examination of the distribution normality was done using the Shapiro-Wilk test. The comparison of two groups was done with the use of Student's $t$-test or Mann-Whitney $U$-test. Data normally distributed are reported as mean \pm standard deviation, and nonnormally distributed measurements are presented as median and minimal-maximal value. Spearman correlation was used to show the relationship of two continuous variables. $P<0.05$ was considered statistically significant.

\section{Results}

The clinical and biochemical data of the studied population are presented in Table 1 . The older group contained $38 \%$ of studied patients and $75 \%$ of them were treated with dialysis. They had a significantly higher renalase $(20.59$ vs $13.14 \mu \mathrm{g} / \mathrm{mL}$, $P=0.02$ ) and dopamine (41.71 vs $15.46 \mathrm{pg} / \mathrm{mL}, P<0.001$ ) 
Table I Clinical characteristic of the study population

\begin{tabular}{|c|c|c|c|}
\hline Parameters & $\begin{array}{l}65 \text { years and } \\
\text { above }(\mathrm{N}=80)\end{array}$ & $\begin{array}{l}\text { Below } 65 \text { years } \\
(\mathrm{N}=131)\end{array}$ & $P$-value \\
\hline Age (years), median (minimum, maximum) & $72(65,86)$ & $52(19,64)$ & 0.001 \\
\hline Body mass index $\left(\mathrm{kg} / \mathrm{m}^{2}\right)$, mean $\pm \mathrm{SD}$ & $29 \pm 4.4$ & $28.32 \pm 4.9$ & $>0.05$ \\
\hline Hemoglobin (g/dL), median (minimum, maximum) & I2.I (7.9, I5.7) & | $3.8(7.2,17.5)$ & 0.001 \\
\hline Cholesterol (mg/dL), median (minimum, maximum) & $176(97,294)$ & $194.0(103,524)$ & 0.037 \\
\hline Triglycerides (mg/dL), median (minimum, maximum) & $106(39,258)$ & $127.5(44.0,575)$ & 0.008 \\
\hline \multicolumn{4}{|c|}{ Echocardiographic parameters, median (minimum, maximum) } \\
\hline Ejection fraction (\%) & $56(16,65)$ & $62.0(30,70)$ & 0.001 \\
\hline Left ventricle $(\mathrm{mm})$ & $4.8(4.2,6.7)$ & $4.85(3.6,6.7)$ & $>0.05$ \\
\hline Left atrium $(\mathrm{mm})$ & $4.0(2.5,5.0)$ & $3.8(2.7,5.3)$ & $>0.05$ \\
\hline Interventricular septum (mm) & $1.2(0.9,1.6)$ & I.I $(0.8,2.3)$ & 0.037 \\
\hline Left ventricle posterior wall (mm) & I.I $(0.9,1.5)$ & I.I $(0.8,1.7)$ & $>0.05$ \\
\hline \multicolumn{4}{|c|}{ Blood pressure values } \\
\hline \multicolumn{4}{|c|}{ Office blood pressure $(\mathrm{mmHg})$, median (minimum, maximum) } \\
\hline Systolic & $139.67(120,190)$ & $140.0(8 \mid, 200)$ & $>0.05$ \\
\hline Diastolic & $75.33(54.7,100)$ & $85(42,120)$ & 0.001 \\
\hline \multicolumn{4}{|l|}{ Additional diseases } \\
\hline Coronary artery disease (\%) & 40 & 13 & 0.001 \\
\hline Diabetes mellitus (\%) & 41.6 & 10 & 0.001 \\
\hline
\end{tabular}

Abbreviation: SD, standard deviation.

concentration (Table 2). Patients aged 65 years and more also had lower diastolic BP (75.33 vs $85 \mathrm{mmHg}, P=0.001)$, advanced abnormalities in echocardiography, such as higher interventricular septum diameter ( 1.2 vs $1.1 \mathrm{~mm}, P=0.03)$ and less ejection fraction rate $(56 \%$ vs $62 \%, P<0.001)$, and more often suffered from diabetes and coronary artery disease. The main used hypotensive drugs in the whole studied cohort were beta-blockers. The older group used more calcium-channel blockers than angiotensin converting enzyme inhibitors. There were no differences between renalase and catecholamine concentration according to the kind of hypotension drugs. A significant correlation between age and the concentration of renalase $(r=0.16 ; P=0.019)$ (Figure 1), norepinephrine ( $r=0.179 ; P=0.013)$, and dopamine $(r=0.21$; $P=0.003$ ) was found in the whole studied population. In the nondialysis subgroup, $44 \%$ had CKD, mostly in stage 2 (83\%). There was a significantly higher norepinephrine concentration ( $1.21 \mathrm{vs} 0.87 \mathrm{ng} / \mathrm{mL} ; P=0.008)$ in older patients of this population. In the dialysis subgroup, there were no differences between renalase and catecholamine level but older people had lower diastolic BP (69 vs $78 \mathrm{mmHg}, P=0.001$ ) and ejection fraction ( $51 \%$ vs $56.8 \%, P=0.03)$. No correlation between renalase and echocardiography parameters was found in the whole study population or subgroups.

\section{Discussion}

Elderly patients compromise the most rapidly growing segment of the US and Europe population and hypertension is highly prevalent among them. According to the study of Egan et al, ${ }^{25}$ two-thirds of older patients suffered from it. Older hypertensive adults experience cardiovascular events at a rate two to three times greater than younger patients with the same BP values. ${ }^{1}$ In the face of antihypertensive treatment benefits in older population, the knowledge of the pathogenesis of hypertension and CVD in elderly is crucial. It is a multifactorial process and one of them is the hyperactivity of sympathetic nervous system. Plasma norepinephrine concentration in older males is $\sim 66 \%$ higher than that observed in the younger due to a significant reduction in norepinephrine clearance from plasma and also an increased sympathetic discharge directed to different organs. ${ }^{2}$ On the other hand, the prevalence of CKD is also fast rising and is highest (up to $38 \%$ ) in patients

Table 2 Renalase and catecholamine concentration in the study population

\begin{tabular}{|c|c|c|c|}
\hline Study parameters & $\begin{array}{l}65 \text { years and } \\
\text { above }(N=80)\end{array}$ & $\begin{array}{l}\text { Below } 65 \text { years } \\
(\mathrm{N}=131)\end{array}$ & $P$-value \\
\hline Renalase $(\mu \mathrm{g} / \mathrm{mL})$, median (minimum, maximum) & $20.59(1.9,62.0)$ & $13.14(0.05,49.9)$ & 0.02 \\
\hline Norepinephrine (ng/mL), median (minimum, maximum) & $0.85(0.2,3.9)$ & $0.79(0.07,2.6)$ & $>0.05$ \\
\hline Dopamine (pg/mL), median (minimum, maximum) & $4 I .7 I(0.8,352.8)$ & I5.46 (90.0I, 363.34) & 0.001 \\
\hline
\end{tabular}




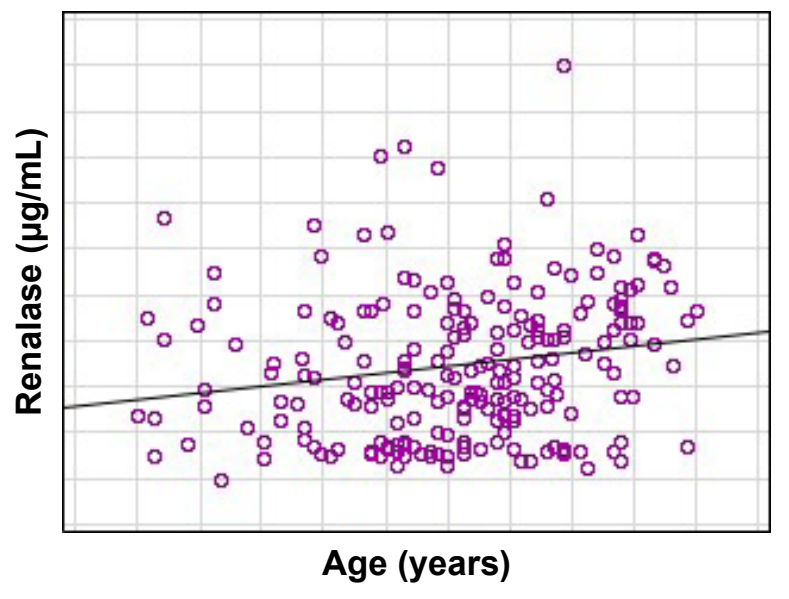

Figure I The correlation between age and renalase in the study population.

older than 65 years, which was also confirmed in our study population. ${ }^{3,26}$ Aging itself is well known to be associated with a progressive decline in kidney reserve. It was found that $30 \%$ of glomeruli were obsolete because of sclerosis in clinically healthy individuals by age $75 .{ }^{27} \mathrm{CKD}$ is also associated with the hyperactivity of sympathetic nerve system which causes the increase of vascular resistance and systemic BP and then leads to CVD. ${ }^{4}$ In our research, the older group more often suffered from end-stage kidney disease and coronary artery disease.

Approximately 10 years ago, a new protein, named renalase, released by the kidney and regulated $\mathrm{BP}$, was discovered. ${ }^{7}$ It was suggested that renalase is a flavin adenine dinucleotide-dependent amine oxidase, which specifically metabolizes catecholamines, with dopamine being the preferred substrate, followed by epinephrine, and then norepinephrine. But the later study by Desir et $\mathrm{al}^{8}$ on 5/6 nephrectomized rats, using in vitro enzymatic assays and in vivo administration of recombinant renalase, showed that it acts as an NADH (the reduced form of nicotinamide adenine dinucleotide)-dependent oxidase that lowers BP by degrading plasma epinephrine. In addition, its enzymatic activity was unaffected by known inhibitors of the flavin adenine dinucleotide-containing amine oxidases, monoamine oxidase-A and monoamine oxidase-B. ${ }^{28}$ The role of renalase in the developing of hypertension and other cardiovascular complications became an interesting topic of animal experiments as well as several human studies. An interesting study was made by $\mathrm{Gu}$ et $\mathrm{a}^{29}$ on the model of heart failure in rats (ligation of the left anterior descending coronary arteryLAD), which showed that renal renalase expression increased and reached a peak at 1-week post-LAD ligation, gradually decreasing in weeks 3 and 4 . They proposed that the kidney might synthesize and secrete more renalase to compensate for the increased catecholamine levels in the early phase of acute myocardial infarction in the animal model. The recent study of Yin et $a^{30}$ made on Sprague-Dawley rats indicated that renalase protected against renal injury and cardiac remodeling after subtotal nephrectomy. The authors concluded that renalase may act as a therapeutic target for the prevention and treatment of cardiorenal syndrome in patients with CKD. Then, in a recent study performed in patients with essential hypertension, the concentrations of renalase, dopamine, and noradrenaline were evaluated compared to healthy individuals and correlated with kidney function. ${ }^{31}$ What is more interesting, it was higher in patients with improper BP control and in those with coronary artery disease and correlated with decreased ejection fraction. However, in a recent study, left ventricular mass index was not related to renalase in the population of 50 hemodialyzed patients. The authors concluded that this correlation may be significant in studies with more patient numbers. ${ }^{32}$ Similarly, we indicated no correlation of renalase with echocardiography parameters of left ventricular hyperthrophy or ejection fraction in our cohort.

There are also genetic studies which evaluate the relationship between renalase and hypertension and CVDs. In one of them, a strong association of the renalase gen polymorphism with stroke in hypertensive patients with and without diabetes was found. ${ }^{18}$ Stec et a ${ }^{19}$ found that renalase gen polymorphism was associated with a higher risk of hypertension in patients with CKD-maintained dialysis. In the most recent meta-analysis, Lv et al ${ }^{33}$ reported that renalase gene rs 2296545 polymorphism is significantly associated with an increased risk of hypertension, whereas rs 2576178 polymorphism may not be associated with the susceptibility to hypertension. On the other hand, the result of the recent study of Kiseljakovic et $\mathrm{al}^{20}$ suggested that renalase gene polymorphism was not a factor that influenced BP in patients on HD. It appears that associations between renalase gene polymorphism and hypertension are still unclear as well as between renalase and CVD. ${ }^{34}$

As the time passed by, more clinical studies using commercially available assays demonstrated that the renalase level was related to the kidney function in just the opposite manner as would be expected from the landmark research of Xu et al. ${ }^{7,11-16,35,36}$ In PD as well as in HD patients, renalase was related to residual renal function. ${ }^{12,13}$ Surprisingly, the Western blot analysis performed in patients after bilateral nephrectomy showed contradictory results to the $\mathrm{Xu}$ et $\mathrm{al}^{11}$ data of the highest renalase level in this group. Gluba-Brzozka et al ${ }^{37}$ found a higher renalase concentration in patients with early stages of CKD than in the control group and supported the previous findings on the relation between renalase and kidney function. In a recent study of Oguz et al, ${ }^{32}$ it was also found that renalase was significantly 
higher in hemodialyzed patients in comparison with the control group. What is also interesting, in kidney transplant recipients, renalase was also dependent on kidney function as well as after heart transplantation. In a recent study of renal transplant recipients with stable graft function, renalase was shown to be a strong predictor of decreased glomerular filtration rate and was significantly higher in the group of patients on cyclosporine, known for the possibility of hypertension and kidney injury developing. ${ }^{38}$ On the other hand, in patients with heart failure due to coronary artery disease or dilated cardiomyopathy, serum renalase level was significantly higher compared to the healthy volunteers. Positive correlation was found between serum renalase and serum creatinine and age. To our knowledge, there are no available data considering renalase and catecholamines in older patients. Our research demonstrated the higher renalase concentrations in older patients with hypertension compared to the younger. It was connected with advanced heart complications and the prevalence of coronary artery disease, which were more often found in older patients. The statistical analysis of correlations revealed the positive one of renalase and age. Approximately $75 \%$ of the older group had $\mathrm{CKD}$ and required dialysis. Interestingly, when we divided the whole study cohort according to maintained dialysis, we found no differences of renalase concentration in patients of different ages in the dialysis and nondialysis subgroups. Older hypertensive persons are a special part of the world population. They are at a higher risk of the development of cardiovascular and CKDs, the disorders strongly associated with an elevated renalase level. According to previous and our current findings, renalase appears to be the possible new marker of the risk of cardiovascular complications and CKD development in older hypertensive patients.

\section{Conclusion}

The elevated renalase level in older hypertensive patients is related rather to kidney function and CVDs than to age itself. Thus, renalase appears to be the possible new marker of these in this special population.

\section{Disclosure}

The authors report no conflicts of interest in this work.

\section{References}

1. Virdis A, Bruno RM, Neves MF, Bernini G, Taddei S, Ghiadoni L. Hypertension in the elderly: an evidence-based review. Curr Pharm Des. 2011;17(28):3020-3031.

2. Xu H, Huang X, Risérus U, et al. Albuminuria, renal dysfunction and circadian blood pressure rhythm in older men: a population-based longitudinal cohort study. Clin Kidney J. 2015;8(5):560-566.
3. Glassock RJ, Rule AD. The implications of anatomical and functional changes of the aging kidney: with an emphasis on the glomeruli. Kidney Int. 2012;82(3):270-277.

4. Zoccali C, Mallamaci F, Parlongo S, et al. Plasma norepinephrine predicts survival and incident cardiovascular events in patients with end-stage renal disease. Circulation. 2002;105(11):1354-1359.

5. RitzE, Rump LC. Control of sympathetic activity - new insights; new therapeutic targets? Nephrol Dial Transplant. 2010;25(4):1048-1050.

6. Domingos F, Escalda A. Causes of autonomic dysfunction in idiopathic recurrent kidney stone formers. Int Urol Nephrol. 2012;44(3): 873-882.

7. $\mathrm{Xu} \mathrm{J,} \mathrm{Li} \mathrm{G,} \mathrm{Wang} \mathrm{P,} \mathrm{et} \mathrm{al.} \mathrm{Renalase} \mathrm{is} \mathrm{a} \mathrm{novel,} \mathrm{soluble} \mathrm{monoamine}$ oxidase that regulates cardiac function and blood pressure. JClin Invest. 2005;115(5):1275-1280.

8. Desir GV, Tang L, Wang P, et al. Renalase lowers ambulatory blood pressure by metabolizing circulating adrenaline. $J$ Am Heart Assoc. 2012;1(4):e002634.

9. Li G, Xu J, Wang P, et al. Catecholamines regulate the activity, secretion, and synthesis of renalase. Circulation. 2008;117(10):1277-1282.

10. Wybraniec MT, Mizia-Stec K. Renalase and Biomarkers of ContrastInduced Acute Kidney Injury. Cardiorenal Med. 2015;6(1):25-36.

11. Zbroch E, Koc-Zorawska E, Malyszko J, Malyszko J, Mysliwiec M. Circulating levels of renalase, norepinephrine, and dopamine in dialysis patients. Ren Fail. 2013;35(5):673-679.

12. Zbroch E, Malyszko J, Malyszko J, Koc-Zorawska E, Mysliwiec M. Renalase in peritoneal dialysis patients is not related to blood pressure, but to dialysis vintage. Perit Dial Int. 2012;32(3):348-351.

13. Zbroch E, Malyszko J, Malyszko JS, Koc-Zorawska E, Mysliwiec M. Renalase, a novel enzyme involved in blood pressure regulation, is related to kidney function but not to blood pressure in hemodialysis patients. Kidney Blood Press Res. 2012;35(6):395-399.

14. Malyszko J, Koc-Zorawska E, Malyszko JS, Kozminski P, Zbroch E, Mysliwiec M. Renalase, stroke, and hypertension in hemodialyzed patients. Ren Fail. 2012;34(6):727-731.

15. Malyszko J, Zbroch E, Malyszko JS, Koc-Zorawska E, Mysliwiec M. Renalase, a novel regulator of blood pressure, is predicted by kidney function in renal transplant recipients. Transplant Proc. 2011;43(8): 3004-3007.

16. Zbroch E, Malyszko J, Malyszko J, Koc-Zorawska E, Mysliwiec M. Renalase, kidney function, and markers of endothelial dysfunction in renal transplant recipients. Pol Arch Med Wewn. 2012;122(1-2):40-44.

17. Li X, Jiang W, Li L, et al. Renalase gene polymorphism in patients with hypertension and concomitant coronary heart disease. Kidney Blood Press Res. 2014;39(1):9-16.

18. Buraczynska M, Zukowski P, Buraczynska K, Mozul S, Ksiazek A. Renalase gene polymorphisms in patients with type 2 diabetes, hypertension and stroke. Neuromolecular Med. 2011;13(4):321-317.

19. Stec A, Semczuk A, Furmaga J, Ksiazek A, Buraczynska M. Polymorphism of the renalase gene in end-stage renal disease patients affected by hypertension. Nephrol Dial Transplant. 2012;27(11): $4162-4166$.

20. Kiseljakovic E, Mackic-Djurovic M, Hasic S, et al. Renalase gene rs2576178 polymorphism in hemodialysis patients: study in Bosnia and Herzegovina. Med Arch. 2016;70(1):31-34.

21. Shi WB, Wang HY. The association study on renalase polymorphism and hypertension: a meta-analysis. Int J Clin Exp Med. 2015; 8(6):9505-9511.

22. Mancia G, Fagard R, Narkiewicz K, et al; Task Force for the Management of Arterial Hypertension of the European Society of Hypertension and the European Society of Cardiology. 2013 ESH/ESC practice guidelines for the management of arterial hypertension. Blood Press. 2014;23(1):3-16.

23. K/DOQI Workgroup. K/DOQI clinical practice guidelines for cardiovascular disease in dialysis patients. Am J Kidney Dis. 2005; 45(Suppl 3):S1-S153.

24. Robinson BM, Tong L, Zhang J, et al. Blood pressure levels and mortality risk among hemodialysis patients in the dialysis outcomes and practice patterns study. Kidney Int. 2012;82(5):570-580. 
25. Egan BM, Zhao Y, Axon RN. US trends in prevalence, awareness, treatment, and control of hypertension, 1988-2008. JAMA. 2010;303(20): 2043-2050.

26. Stevens LA, Li S, Wang C, et al. Prevalence of CKD and comorbid illness in elderly patients in the United States: results from the Kidney Early Evaluation Program (KEEP). Am J Kidney Dis. 2010;55(3 Suppl 2): S23-S33.

27. Qian Q, Nasr SH. Diagnosis and treatment of glomerular diseases in elderly patients. Adv Chronic Kidney Dis. 2014;21(2):228-246.

28. Severina IS, Fedchenko VI, Veselovsky AV, Medvedev AE. The history of renalase from amine oxidase to a-NAD(P)H-oxidase/anomerase. Biomed Khim. 2015;61(6):667-679.

29. Gu R, Lu W, Xie J, Bai J, Xu B. Renalase deficiency in heart failure model of rats-a potential mechanism underlying circulating norepinephrine accumulation. PLoS One. 2011;6(1):e14633.

30. Yin J, Lu Z, Wang F, et al. Renalase attenuates hypertension, renal injury and cardiac remodelling in rats with subtotal nephrectomy. J Cell Mol Med. 2016;20(6):1106-1117.

31. Maciorkowska D, Zbroch E, Malyszko J. Circulating renalase, catecholamines, and vascular adhesion protein 1 in hypertensive patients. J Am Soc Hypertens. 2015;9(11):855-864.

32. Oguz EG, Gursoy GK, Yayar O, et al. Increased serum renalase in hemodialysis patients: is it related to left ventricular hypertrophy? Ren Fail. Epub 2016 Jul 14:1-7.
33. Lv YB, Wang Y, Ma WG, et al. Association of renalase SNPs rs 2296545 and rs2576178 with the risk of hypertension: a meta-analysis. PLOS One. 2016;11(7): $\mathrm{e} 0158880$.

34. Musiałowska D, Małyszko J. Renalase - new marker or just bystander in cardiovascular disease-clinical and experimental data. Kardiol Pol. Epub 2016 Jun 14.

35. Dziedzic M, Petkowicz B, Bednarek-Skublewska A, Solski J, Buczaj A, Choina P. Relationship between renalase and N-terminal pro-B-type natriuretic peptide (NT pro-BNP) in haemodialysis patients. Ann Agric Environ Med. 2014;21(1):132-135.

36. Przybylowski P, Malyszko J, Kozlowska S, Malyszko J, Koc-Zorawska E, Mysliwiec M. Serum renalase depends on kidney function but not on blood pressure in heart transplant recipients. Transplant Proc. 2011; 43(10):3888-3891.

37. Gluba-Brzozka A, Michalska M, Franczyk-Skora B, Nocun M, Banach M, Rysz J. Markers of increased cardiovascular risk in patients with chronic kidney disease. Lipids Health Dis. 2014;13:135.

38. Stojanovic D, Cvetkovic T, Stojanovic M, Bojanic V, Stefanovic N, Stojanovic I. The assessment of renalase: searching for the best predictor of early renal dysfunction by multivariate modeling in stable renal transplant recipients. Ann Transplant. 2015;20:186-192.
Clinical Interventions in Aging

\section{Publish your work in this journal}

Clinical Interventions in Aging is an international, peer-reviewed journal focusing on evidence-based reports on the value or lack thereof of treatments intended to prevent or delay the onset of maladaptive correlates of aging in human beings. This journal is indexed on PubMed Central, MedLine,

\section{Dovepress}

CAS, Scopus and the Elsevier Bibliographic databases. The manuscript management system is completely online and includes a very quick and fair peer-review system, which is all easy to use. Visit http://www.dovepress. com/testimonials.php to read real quotes from published authors. 